\title{
Self-Organization of Cognitive and Interactional Processes
}

\author{
Jürgen Kriz \\ University of Osnabrück, Dept. of Psychology * Psychotherapy and Clinical Psychology \\ e-mail: kriz@uos.de \\ homepage: $\underline{w w w . j k r i z . d e}$
}

Key words:

pattern formation, pattern recognition, self-organization, completion dynamics, cognition, interaction, teleology

\begin{abstract}
In the last decades new concepts have emerged in order to understand pattern formation in cognitive and interactional processes. Some of these concepts are „emergence“, ,attractor“ and „,selforganization“. Many „old“ phenomena, well known in psychology (for example in Gestalt Psychology) - such as „Gestalt“, „,tendency towards praegnanz“, ,schema“, „stability and changes of attitudes", interaction patterns in couples and families, etc. can be understand and reconstructed within the frame of these concepts. Especially the interdisciplinary field of "Synergetics“ by H. Haken has turned out to serve as a fruitful approach and source for may research projects even in the field of psychology (to which cognitive processes belong, too). Based on the exploited pronounced analogy between pattern formation by self-organization and pattern recognition, especially the completion of an incomplete set of data (due to the dynamical structure of an attractive process) is of interest.

The text will report some basic concepts and outline some questions, approaches and experimental findings. Finally, I present a plea for a reassessment of and a re-esteem for the teleological principle in order to investigate and understand the ordering forces in human's world.
\end{abstract}

\section{Introduction}

Focusing on the relationship between systems science and psychology (or social science, respectively), there are two different perspectives: (a) the contribution of (to a great extent: "classical") psychology to systems science in order to increase the abilities of humans in dealing with "systems" and (b) the contribution of systems science to psychology in order to understand and describe psychological (and social) processes in a more profound way.

(a) Under the former perspective, researchers in non-psychological fields are interested, for example, in ecosystem theories concerning energy use, dissipation processes, industrial strategies, matter-energy-flows and emissions, etc. However, when theoretical assumptions and computer models are applied to the "real world" researchers are often disappointed about the outcomes: Problems turn out to be ill-posed due to the fact that humans do not behave in that way which the rationality of mathematical equations and of models from the natural sciences say that they should do in order to minimize costs, energy, etc. Nuclear power stations are not only questioned about due to their physically and mathematically computed security and due to the disposal of nuclear waste, energy, economics, and of other "objective" parameters. It is more a question of how to deal with and respect human's fears, images of danger, the believe in human errors and acts of sabotage, and so on. From the bottom of this experience, the demands which systems scientists make on the contribution of psychology are rather "classical" ones and can be described by terms such as "education", "training", or "counseling". The leading question is: "how do people interact with complex systems ?" - 
where the term "system" does not only represent the environment in an ecological sense but also means large organizations, where a special competence in dealing with gate keepers and other important persons is necessary. The aim is to understand and overcome the „,irrationality“ of behavior and, moreover, the gap between knowledge and acting. This leads to research on and the practice of management training, counseling of organizations and so on, and, moreover, in research and training of ,system-competence“ by way of, for example, analyzing the behavior of persons when they have to act with respect to computer-simulated systems with all the contra-intuitive behavior of non-linear system dynamics.

(b) On the other hand, researchers are interested in the contribution of systems theory in order to deal with questions such as: "how can we understand and influence patterns of perception, thinking (e.g. "schemata"), reasoning, and imagination, and, moreover, patterns of interaction in families and groups?" These questions refer to concepts such as "self-organization", "emergence", "phase-transition", "(in)stability" and so on. The treatment and discussion of these questions have been influenced to a great deal by the concepts and the interdisciplinary approach "Synergetics" - initiated and coined by the German physicist Hermann Haken (see Haken 1983). Though synergetics originated from physics (especially laser theory), its mathematical and conceptual formulations soon turned out to be applicable to a variety of fields because synergetics deals with self-organizing complex systems in a very general manner. Solely the "Springer Series in Synergetics" comprises nearly one hundred volumes with about 2.000 contributions from may different disciplines - of course, mostly from the natural sciences, however, increasingly, also from psychology, cognitive and social sciences (see, for example, Haken 1988, 1992, 1996, Haken and Stadler 1990, Tschacher et al. 1992, Tschacher and Dauwalder 1999). By means of the interaction of their components complex systems can produce new qualitative features on macroscopic scales. The central aim of synergetics is, therefore, to describe general principles that govern the behavior of complex systems when these qualitative changes take place, i.e. when a system leaves a certain state (a structure or pattern of the dynamics) and - by passing a gate of instability - starts a new structure or behavior.

This is, of course, not only typical and important for phenomena in the natural sciences but also for psychological, social and cognitive processes: For example, many authors in different sub-disciplines refer to patterns of communications or interactions in small (families) or bigger (organizations) groups questioning why these patterns do or do not change and what could be done in order to stabilize or to change. On the other hand, by focusing on interactions only, one may become blind for the fact that the persons who have come together have brought certain experiences and habits into this relationship, which have an effect on their setting up certain rules. "Patterns of the interactions", therefore, also reflect patterns of expectations, interpretations, definitions of "reality", etc. by the group members - and the same questions about stability, instability, conditions for phase transitions etc. may arise. Psychotherapy, for example, can be understood, from this perspective, as a phase transition of a "pathological" interactive and/or cognitive pattern to a "better" one (whatever this may mean in detail). As a consequence, we find a hierarchy of phenomena, each level may be described in terms of selforganized processes: Not only patterns of interaction are the results of self-organized processses but, by the same logic, thoughts, cognitions, processes of the central nervous system and, on the other hand, society and several sub-systems of the society can be understood as selforganized processes, too.

Undoubtedly, the perspective (a) is an important and fruitful one (see, for example, the research of Ceccini 1988, Dörner and Wearing 1995, Kim 1994, Klabbers 1984, W.C. Kriz 1999, Meadows and Toth 1985, Senge 1990, or Thiagarajan 1993). However, in the following 
I will discuss some aspects and research more from the latter perspective (b). Especially in the German speaking countries, the synergetic concepts of self-organization had an immense impact on research in psychology - among others, papers by E.J. Brunner, G. Schiepek, W. Tschacher, M. Stadler and myself have been reported in many books and the former three persons have organized fall-symposia nearly every year in the last decade (see Tschacher et al. 1992), recently extended to "Joint Conference on Complex Systems in Psychology" in 1997 and 2000 together with researches from the US (see Tschacher and Dauwalder 1999).

\section{The Contribution of Gestalt Psychology}

However, despite of this perspective - i.e. the contribution of systems science to psychology - it should be noted that a great deal of concepts which are discussed nowadays in the frame of modern systems science (and of which most natural scientists believe that these notions emerged in the field of the natural science in the sixties of the $20^{\text {th }}$ century and later) have been already investigated theoretically and experimentally in the field of psychology at the beginning of the $20^{\text {th }}$ century. Especially the famous Berlin school of "Gestalt Psychology" (famous names among others are Max Wertheimer, Kurt Koffka, Wolfgang Köhler, Kurt Goldstein, Kurt Lewin) did a lot of fundamental research between 1910 and about 1940 (when all these researchers had to leave Germany due to the Nazi-regime) and inspired many other researchers all over the world. In gestalt psychology the autonomy of order formation in cognitive processes was the explicit starting point of conceptualization and experimental work. The concept of gestalt quality is defined as emerging from elementary interactions but not completely reducible to them: The parts often derive their nature and purpose from the whole and cannot be understood apart from it. Moreover, a straightforward summation process of individual elements cannot account for the whole - a "melody" has another quality than the sum ore sequence of "tones": The "Gestalt" of a melody can be transposed and will be detected as "the same melody" even if the transposed melody contains not one single tone of the old melody. Moreover, new features arise - even addressed to tones (e.g. the "tonic" or "key note" and other musical concepts). With respect to the process of perception, Gestalt psychologists stressed the fact that activities within the total field of the whole govern the perceptual dynamic. By three decades of research, Gestalt psychology has formulated a lot of fundamental principles of order formation. The most powerful of them is the tendency towards "Prägnanz" (which can be translated from the German approximately as "salience").

In addition, also topics in social psychology have been studied from the structuralist Gestalt viewpoint, as in Kurt Lewin's work on group dynamics. With respect to modern systems science Kruse et al. (1992, p 103) states: "There are astonishing correspondences between the terminology and the argumentation of gestalt theory and modern self-organization concepts. In particular the basic assumptions of Wolfgang Köhler are nearly identical to modern approaches." And the physicist Haken (1992, p 44) stresses: "One of the prominent features of synergetics is the consideration of the emergence of new qualities ... This was clearly put forward by the Gestalt psychologists, who, at their time, had to fight structuralism, in which the attempt was made to explain the properties of the whole out of the properties of the individual parts.... Other concepts found in Gestalt psychology are those of bistability of perception ... and of hysteresis" - two concepts which we are going also to refer to a little later.

As a consequence, the "old" concepts of gestalt psychology are reassessed nowadays. For example, in his book "The Organism" (Germ. 1933, Amer. 1939) the Gestalt psychologist and physiologist Kurt Goldstein coined the term "self-actualization" (which later became a central 
term also in the therapeutic approach of Carl Rogers) and "self realization". In this old book one finds phrases which sounds impressive modern, like: "the organism is an organized system ... it is impossible to understand the whole by directly studying isolated parts and segments because the whole function cannot be found in the parts". Moreover, Goldstein stressed that his approach - which became in the United States more famous under the name "Organismic Theory" - does not require an ,organizer" because organization is built into the system from the beginning and the integrity of the organism is not permitted to be lost or destroyed by analysis. Although organismic theory does not regard the individual as a closed system, it tends to minimize the primary and directive influence of the external environment. He also made very clear, that from this perspective development has to be understood as "reorganization of old patterns into new and more effective patterns" (what we, nowadays, would refer to by the term "phase-transition"). Moreover, the seeming "modern" term "biosphere" has been coined already 1941 in the work by Andras Angyal, another researcher in the field of Gestalt psychology.

Finally, already in 1932, in his book "Remembering", Sir Frederic Bartlett introduced the idea of serial reproductions in order to investigate the dynamic of remembering. He used recursive iteration procedures as a methodological tool for the demonstration and analysis of order formation. The basic idea is to ask a person to reproduce a given visual or cognitive pattern (for example, a short story) and then to use this reproduction as a stimulus for the next reproduction (by another or by the same person) and so on. If the pattern is very complex (for example a random configuration or an ambiguous pattern) it may change from iteration to iteration due to the fact that it cannot hold in mind completely. However, one often find that this process tends to highly ordered structures which are steady states - or, in the terminology of systems dynamics, "attractors": Due to the ordering forces of the cognitive system the pattern changes less and less until it is no more subject to alterations in further reproductions. In every day life this procedure is well known in the field of rumour generation. Such serial reproductions may be regarded as data for a detailed analysis of slight systemic tendencies in the self-organization process in perceptive and cognitive systems. While Bartlett's methodology has been used, for example, in the linguistic analysis of text understanding and remembering short stories, Stadler and Kruse (1990) reported a perceptual study in which they showed that serial reproductions of random dot patterns tend to patterns of high "Prägnanz" (see figure 7 in the chapter of Stadler and Haynes in this volume).

\section{Iterative Designs}

In contrast to analyze order formation processes in visual perception by way of the Bartlett methodology, in Osnabrück we used the serial reproductions in order to investigate psychological processes on a "higher" level. According to Bartlett's interests concerning the structuring forces in the dynamics of remembering we used descriptions of persons (for example, by lists of features) in order to demonstrates the pattern formation in remembering (or constructing, inventing) the "personality"of a person. In one study (Kriz and Kriz 1992) 30 subjects were given 72 statements which describe a fictive person. These statements were very short because the just combined 72 pairs of adjectives ("intelligent-unintelligent", "happyunhappy", "friendly-unfriendly", etc.) with 4 qualitative categories ("extreme", "very", "rather" "little"). Accordingly, the statements were like: Person X is "very intelligent", "extreme unhappy", "rather friendly" etc. A subject had to read these 72 statements in as much time as he wanted. After some minutes of doing mental arithmetic (in order to have some other cog- 
nitive activity) he was asked to reproduce the statements by making crosses in a list with 72 pairs of adjectives - each pair in one line with a nine-point-scale between $(2 \times 4$ categories plus "I don't know").

These answers were typed into a computer while the subject again was doing some mental arithmetic. The next step then was to present the subject a printout of his answers - however, transformed again in statements and printed out by the computer. This procedure was repeated 10 times.

In figure 1 the ordinate represents a measure of change from one cycle to the next one (which is just the mean of all differences) while the abscissa shows the 10 cycles of serial production ("0" is the initial material). The solid line is computed from the differences between "input" and "output" in each cycle. Accordingly, a difference of 0 (or nearly 0 ) stands for the fact, that the description of the person is perfect (or nearly perfect) repeated. In Fig. 1 this is the case after 7 cycles while at the first cycles we find rather big changes (a maximum change would be at about 6 because from one extreme "-4" to the other " +4 " is the difference 8 , but if we start from "-1" the maximum possible difference is 5).

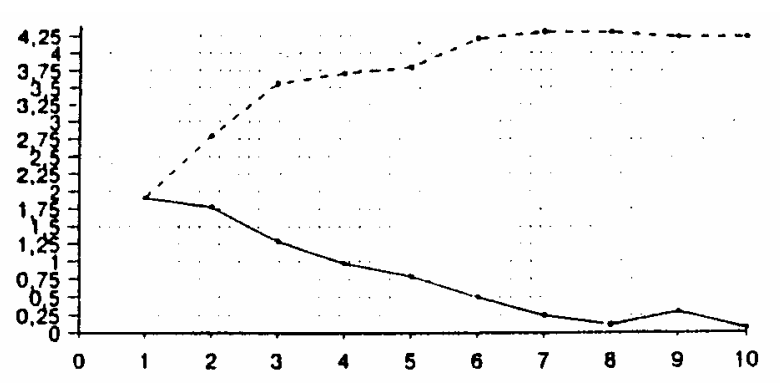

Fig. 1: Dynamics of mean differences between two successive serial reproductions (solid line) and between the reproductions and the initial description of attributes. Data from one typical subject.

It is evident that the process of serial descriptions of the person has an attractive dynamic. It is important to note that Fig. 1 is typical to 25 out of 30 subjects. However, for 5 subjects no attractive dynamic could be seen (in 10 cycles): the differences between successive cycles or, what is the same: between "input" and "output" - stayed to be rather big.

Another important point to be made about the attractive dynamic (by these 25 subjects) is the fact that the descriptions of persons attitudes became rather quick stable, but these stable "descriptions" turn out to be merely constructions: The dotted line in Fig. 1 represents the mean differences of each cycle to the initial description. It couldn't be more dissimilar!

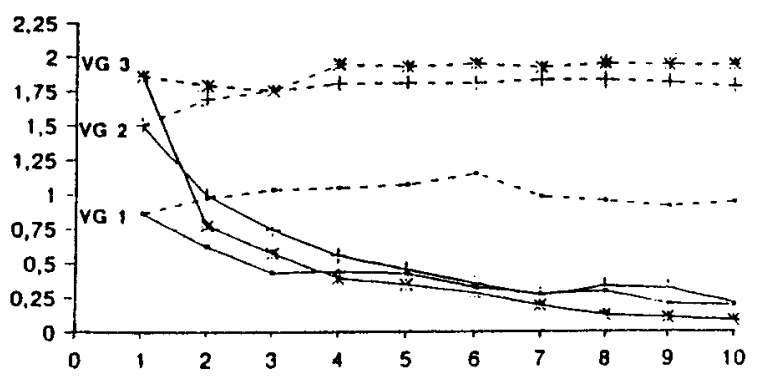

Fig. 2: Mean dynamics (comp. to Fig. 1) for three groups: VG 1 rather consistent, VG 2 little more inconsistent, VG 3 rather inconsistent initial description of attributes 
A last finding from this study may be mentioned here: 3 different conditions were given to the 30 subjects (each condition to 10 subjects): The initial description was (i) rather consistent (ii) medium consistent and (iii) rather inconsistent (for example, the person was described as "very friendly" but later as "rather gruff"). While differences between successive cycles became rather fast 0 (or nearly 0) in all 3 conditions (for 25 subjects) the differences in the first two cycles were significantly higher for the inconsistent description than for the medium consistent description and were significantly lowest for the consistent description (see Fig. 2). Of course, the total difference of the stable pattern from the initial pattern follows shows the same effects. In conclusion it may be stated that constructive pattern formation/recognition changes an inconsistent "reality" more than an consistent "reality". This is rather likely because we all want to live in a consistent cognitive world rather than in a inconsistent one.

In other experiments (Kriz, Kessler and Runde 1992) the process of pattern formation by use of personality inventories was investigated: Subjects were given 10 statements of a German personality inventory (FPI-R) - which is a very rough, vague and colorless information. The subjects were told to assume that these ten items are statements made by "Mr. K. from W." about himself when they had "met him during their holiday in Spain". The subjects then got a questionnaire with 200 more items from that personality test. They were asked to guess whether "Mr. K. from W." would have agreed to that statement about him. Additionally, they were asked to scale how sure they are in their judgment on a rating scale between 1 and 5 . These 200 items were presented on 20 pages of paper, 10 items/page. Moreover, each page with 10 items represented a random sequence of exactly the 10 personality dimensions. As a consequence, this procedure can again be understood as a serial production procedure with 20 cycles, 10 items each.

One interesting result of that study is the fact, that all "normal" subjects show attractive guesses only in some dimensions while in other dimensions random guesses indicates that no notion of Mr. K. has been formed. Moreover, these dimension vary from subject to subject. In contrast, "clinical" subjects - patients of a psychiatric hospital with the diagnosis "schizophrenic disease" but not in an acute phase or attack of symptoms - did not show any stable guessing at the personality scales. They were clearly unable to form a pattern of the personality of Mr. K. (for details see Kriz 1993).

\section{Pattern Formation, Pattern Recognition and Completion- Dynamics}

These experiments of pattern formation can also be discussed in the frame of the analogy between pattern formation and pattern recognition which has been conceptually invented and studied by the Haken and his group with respect to the synergetic computer. Figures 3 and 4 demonstrate the fundamental principle of this analogy between pattern formation and pattern recognition: When part of the subsystems are in an ordered state, they generate their order parameter which then "enslaves" the rest of the system and produces a totally ordered pattern (Fig.3, left); on the other hand, when some features of a pattern are given, they generate their corresponding order parameters which then restores the whole recognition pattern (Fig.3, right). Following this principle and the mathematical procedures (which are in some details 
represented in Haken 1992) Fig. 4 shows a visualization of the pattern recognition procedure. The synergetic computer recognizes a face and its name (a letter) out of an initially given set of features - (a): a strong superposition of noise on it; (b) just a small part of it.

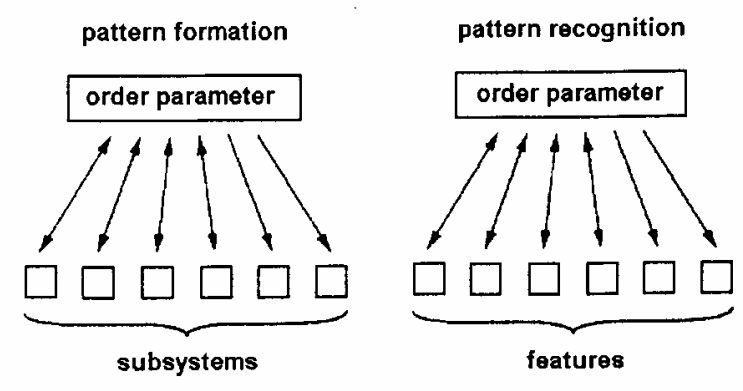

Fig. 3: The analogy between pattern formation and pattern recognition (compare text)

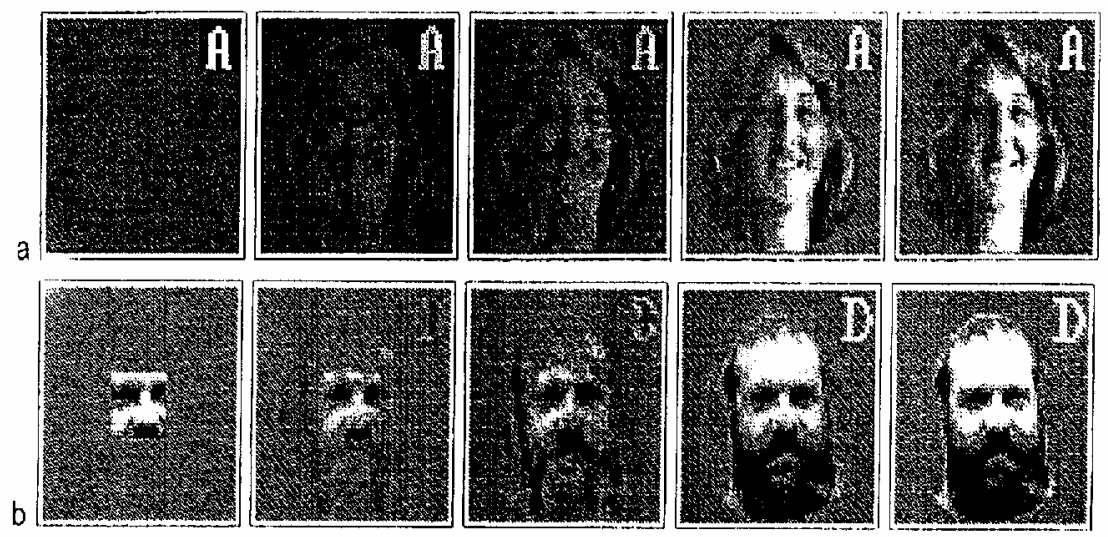

Fig. 4: Visualization of the pattern recognition procedure (After Haken 1992)

Of course, the synergetic concept of pattern formation and pattern recognition is nice in the case of a synergetic computer where noisy or distorted patterns have to become recognized. In contrast, if this is a model for what happens in associative memory - and there is a lot of evidence for it - then parts of a set of data is "completed" in a unique fashion. However, this might not only happen when we "recognize" the face of an old friend from a short visual impression, or a word from some letters, or the context of a situation from some pieces of information. By the same logic, we might generate and construct patterns on the basis of very little or unclear information. And, what is even more important, while we believe we restore or recognize the information we are just constructing this (part of our) reality - due to order parameters, which might have emerged decades earlier (for example, in the early childhood, when not only the order parameters of our language - grammar - are "learned" and, more generally, the order parameters of the semiotic language of our symbolic world but, even more generally, many order parameters of structuring our personal world (see Kriz 1997).

Even in his early work Bartlett (1932) stressed the point that a schema is ,an active organization of past experience“ and, additionally, ,together with the immediately incoming impulse it renders a specific adaptive reaction possible“ and, in addition, that „remembering appears to be far more decisively an affair of construction rather than of mere reproduction". It is quite likely that this, in the social area, also happens in cases of prejudice, stereotypes etc. Therefore, in the area of human systems, the completion dynamic of ambiguous or "lousy" 
information to a complete "image" is very typical - however, it should be noted that in many cases this "image" does not images any "true" structure or picture (as the synergetic computer does) but that the small information pieces might be no more than just a trigger for the completion dynamics in which we "fantasize" or "hallucinate" an order (due to inner order parameters) of which we believe that this would be the order of something from "outside" (for example, the shape of a face, the meaning of a given comment, or the "personality" of another person).

Again an experiment conducted more than 50 years ago by Salomon Asch (1946) can illuminate that point how people form impressions of others' personalities. The experimenter read to his subjects a number of discrete characteristics which were said to apply to a fictitious person. One such list, for example, was ,intelligent, industrious, impulsive, critical, stubborn, envious“. Asch then instructed his subjects to write a brief description of the impression they had of the unknown person. The results demonstrated that the discrete terms of the list had been organized into a meaningful and even colorful personality. Moreover, another group was given the same terms, but in reverse order. As a consequence, whereas the former subjects had described the fictitious person in a positive manner, the latter described him in a negative manner. This effect has been called the primacy effect. However, we can interpret these findings within the scope of completion dynamics (or the dualism of pattern formation and pattern recognition), because the first items of a list undoubtedly started a completion dynamics which let an image of a person emerge (still rough and vague but nevertheless more complete than just one single characteristic) which again ordered the interprettation process that enslaved the meaning of the following terms in a positive (or negative) manner. For example, the understanding of the term 'stubborn' is governed by the order parameters of a positive schema of the unknown person (see Fig. 5 with respect to Fig. 3). As a philosophical remark, we can even understand the famous "hermeneutic circle" - the interpretative way to find out the meaning of a given text or semiotic object - in this frame of this iteration-process of completion dynamics and pattern recognition (or pattern formation, respectively).
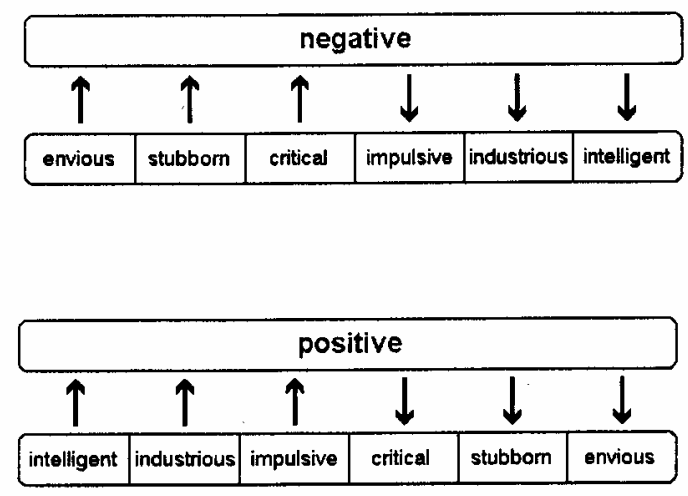

Fig. 5: Pattern formation and pattern recognition in the experiment of Ash

In a recently conducted experiment (carried out by Kay Wegner) we presented test persons a "story of a man's day" in which ten positive and ten negative events occurred (of course, in a random sequence). All Ss got the same story but the first sentence was different: some Ss got the information that it was a rainy day when that man woke up while some other Ss got the information that the sun was shining. Moreover, we had a group of depressive Ss and another group of non-depressive Ss. There were a lot of specific effects in remembering and retelling this story: Depressive persons reproduced significantly less positive than negative events un- 
der the condition "rainy day" while non-depressive Ss showed that difference under the condition "sunny day". Moreover, depressive Ss judged the whole story much more often in an negative manner under the condition "rainy day" than under the other condition (no differences were found for non-depressive Ss). These and some other results have to be replicated however, they support at least our central message that further research from that perspective seems to be of great interest in order to understand the structuring forces in the every-dayreality.

In another experiment (carried out by Mareike Lang) we used the serial reproduction technique to investigate the reduction of information by pattern formation with respect to two fictive persons (instead of only one). Ss were given the description of two persons A and B by way of 32 attributes. 16 of these attributes came from a pretest in which each subject developed his own categories in order to describe relevant persons (mother, father, partner). Then the subject was asked to describe the fictive person A (and then B) by making crosses in a list with 48 pairs of adjectives ( 32 were the starting attributes). Like in the first reported experiments, the next step then was to present the subject a "further" description of A and B based on his own answers. This procedure was repeated 10 times. With respect to A and B only we found the same results as in the above reported experiment: The repetition of the description became rather stable after some steps - again, however, far away from the initial description. In addition, to manage the problem of too much information concerning two fictive persons $\mathrm{A}$ and B, most Ss constructed a big contrast between A and B. For example, while the initial description had 18 positive and 14 negative adjectives both for A and B Ss constructed description after some steps in which one person had a proportion of 3:1 (or even higher) of positive/negative adjectives for A and vice versa for B.

All these findings support the conception of attracting processes which bring order to complex material by way reduction and stabilize these patterns - and, by the same way, let expect clear effects of hysteresis. In analogy to a switching process in physical systems the term "hysteresis" refers to the fact that the state or pattern of a system depends on its history: due to the attracting forces a phase transition from state (a) to state (b) by changing the conditions (for example the given information) will not take place at the same point (referring to the control parameter(s) or these conditions, respectively) where we will found the transition from (b) to (a) but will stay longer in the former state and resist longer to turn into the new pattern or state. When we slightly change a circle into a square on a computer screen, the same configuration(s) somewhere in the middle of that line we will still be seen to be a circle while we still will see a square when we start with a square changing into a circle.

In order to investigate hysteresis effects in the process of personality attribution we conducted some experiments. As expected, we found some hysteresis, however the results are not so clear until now. Firstly, there are big differences between the individuals. Secondly, people seem to be very flexible in changing their cognitive patterns for the first change of information and then, for the second and third change (we didn't have more), stay rather stable (which would be a strong hysteresis). The reason for the unexpected flexible reaction and transition to new "conditions" (information) might be explained by the fact that under typical experimental conditions the test persons are highly motivated to respond to any information given by the experimenter.

In one part of the above outlined serial reproduction experiments (carried out by Mareike Lang) the Ss got some new (and inconsistent) information before the $4^{\text {th }}$ and the $7^{\text {th }}$ step (of 10 steps): before the $4^{\text {th }}$ step 16 of the initially given 48 attributes were given as "additionally offered information" about the (only one) person A however in strong contrast to the initial description; before the $7^{\text {th }}$ step again 16 attributes were "additionally" given - this time very 
consistent to the first description (but in contrast to the information at step 4). Ss reacted very prompt and strong to the first change but, in contrast, very little to the second one.

In another experiment (carried out by Markus Pohl) Ss were given a detective story concerning the death of a man caused by his girl friend who smashed a bottle on his head to pieces so that he died. The story was presented bit by bit (or better: sentence by sentence) on a computer screen - and after each sentence the Ss had to judge about the case by means of some questions and given scales (from 0 to 100 between two opposite terms). The most important judgment was the decision "mad" (the girl has to be brought into a psychiatric hospital) or "bad" (the girl has to be sent to prison). After a neutral information one group of Ss got 6 statements which gave support the "mad"-hypothesis, followed by 8 "bad"-statements, then again 4 "mad"-statements and, finally, 2 "bad" statements (so that the sum amounts to 10 "bad"- and 10 "mad"-statements). Another group of Ss got the opposite sequence (6 "bad", 8 "mad" etc.).
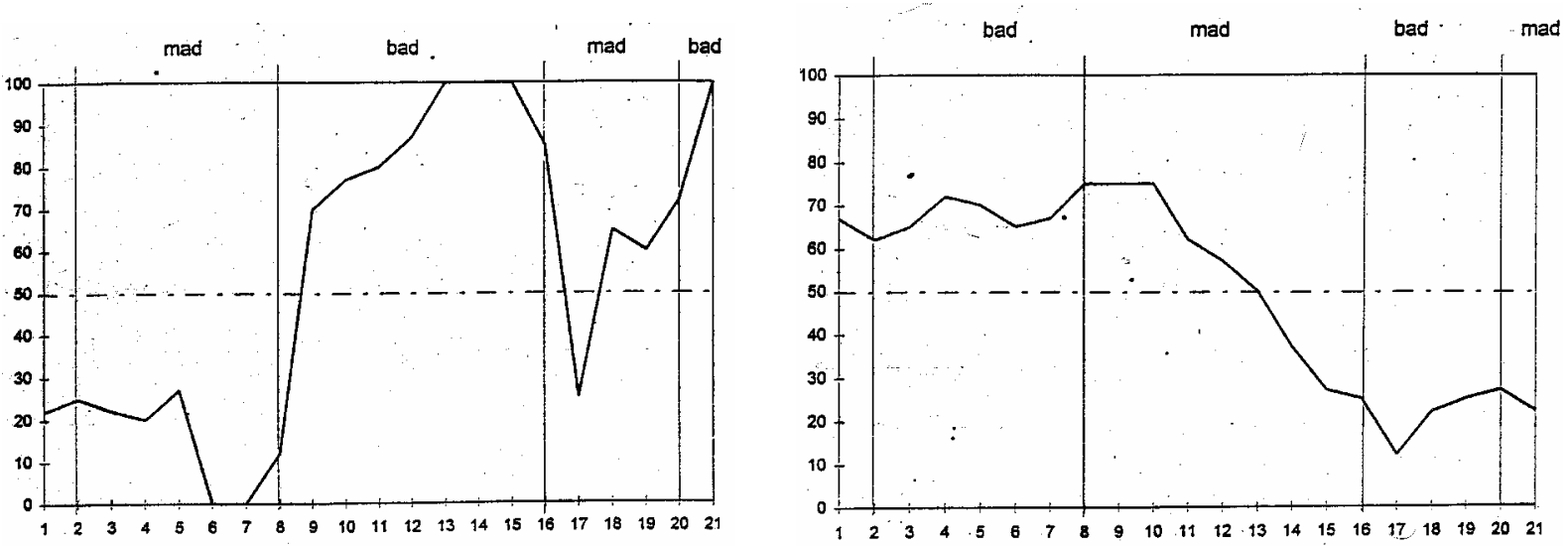

Fig. 6 a, b: Judgments of "bad" (criminal - ratings > 50) or "mad" (insane - ratings < 50) due to "mad"- / "bad"-information-pieces of a detective story. Two different subjects.

Fig. 6a and $6 \mathrm{~b}$ show the sequences of reactions of two different Ss (values over 50 mean "bad", under 50 "mad") and indicate the big individual differences in reacting. However which is typical for most Ss - one can see the big influence of the first change (from "mad"statements to "bad"-statements or vice versa, respectively) which reverses the judgment, while after the second change of statements the judgment stays (mostly) in the same direction. This is true for different groups of students and other persons - despite of the fact that there were plain (but expected) differences in the way that law students judged much higher on the scale "bad" (in contrast to "mad") throughout the information steps than did students of psychology and a mixed group of students of different other subjects.

\section{Patterns of Interaction}

At the beginning, we have stressed the point that patterns of the interactions also reflect patterns of expectations, interpretations, definitions of "reality", etc. by the group members due to the fact that the persons who have come together have brought certain experiences and habits into this relationship, which have an effect on their setting up certain rules. In the "person-centered system approach" (Kriz 1991) I have stated that patterns or rules in the communication process of a family is related to categories of perceptive and cognitive processing of information (e.g. communications of other family members) - especially in families where ob- 
servers describe a "lack of flexibility" and a "rigid" behaviour. Therapist often hear the argument of single family members: "Why should I change my behavior - the others wouldn't notice it". This seems to demonstrate that only a few pieces of information are taken and may already suffice as the basis for pattern formation or "recognition" - that means that the order parameters of the cognitive patterns (=interpretation of the behaviour) follow a trajectory towards an attractor, which might be of low dimensionality and, moreover, there may exist only very few attractors. If a family member experiences only very few categories (attractors) of behaviour, motivations etc. there is no reason to "react" to these in a high differentiated manner. As a consequence, he or she will practice a small repertoire of communication behaviour. As in the whole communication process of a family the communication behavior of one person is closely related to the perception-, cognition-, and interpretation-process of an other one - and vice versa - this dynamic may show a decreasing repertoire both in "expressions" and "impressions" of communication behavior.

In order to analyze the described process empirically we conducted some experiments where we brought pairs of persons (for example a female and a male student) together - strictly noting that they did not know each other - and asked them, for example, to solve problems together. Additionally, we asked them to give feed back to each other (on given scales) in order to start feed-back-loops that will not only lead to individually stabilized patterns (of judging the partner) but also lead to patterns of interaction.

In one of these experiments (carried out by Matthias Ochs) we asked pairs of students to judge a given picture of a human's head - which, however, was only shadowy presented (the pixels were superimposed by a big amount of noise). The judgments had to be done on a standard instrument for process-oriented interaction analysis (System for Multiple Level Observation of Groups, SYMLOG, by Bales and Cohen 1979). Again, the experiment was conducted in 9 steps: at each step each person of the pair made his judgments on a sheet of paper and then read these loud so that the other could listen. No further discussion was allowed. In addition, the students were also asked to judge (on a similar scale) at each step (a) themselves, (b) the other person, (c) how they believe that the other person would judge them - however, these three judgments were not read out to the other.

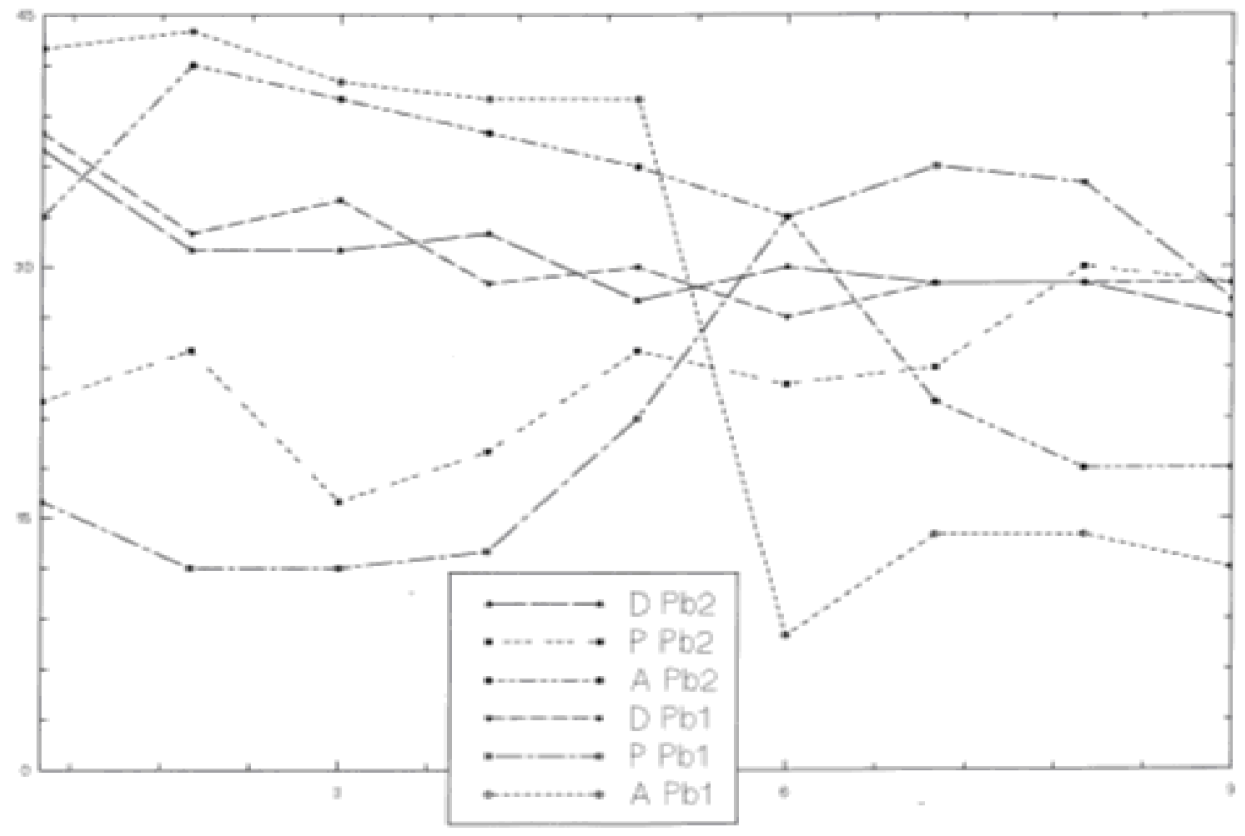

Fig. 7: Phase transition in the SYMOLOG ratings of 2 Ss over 9 cycles 
Although in some pairs did not happen anything of interest (as far as we could see it in the data, of course) there were a lot of interesting phenomena in other. Without going into further details here - and only on a phenomenological level - in the last third of Fig.7 we can see something like a phase-transition of the judgment-pattern concerning the three SYMLOGdimension $\mathrm{D}, \mathrm{P}$ and $\mathrm{A}$ and the student-pair $\mathrm{Pb} 1$ and $\mathrm{Pb} 2$ (it would go beyond the scope of this paper to explain the dimensions $\mathrm{D}, \mathrm{P}$ and $\mathrm{A}$ in an understandable manner because there is a lot of conceptual work behind).

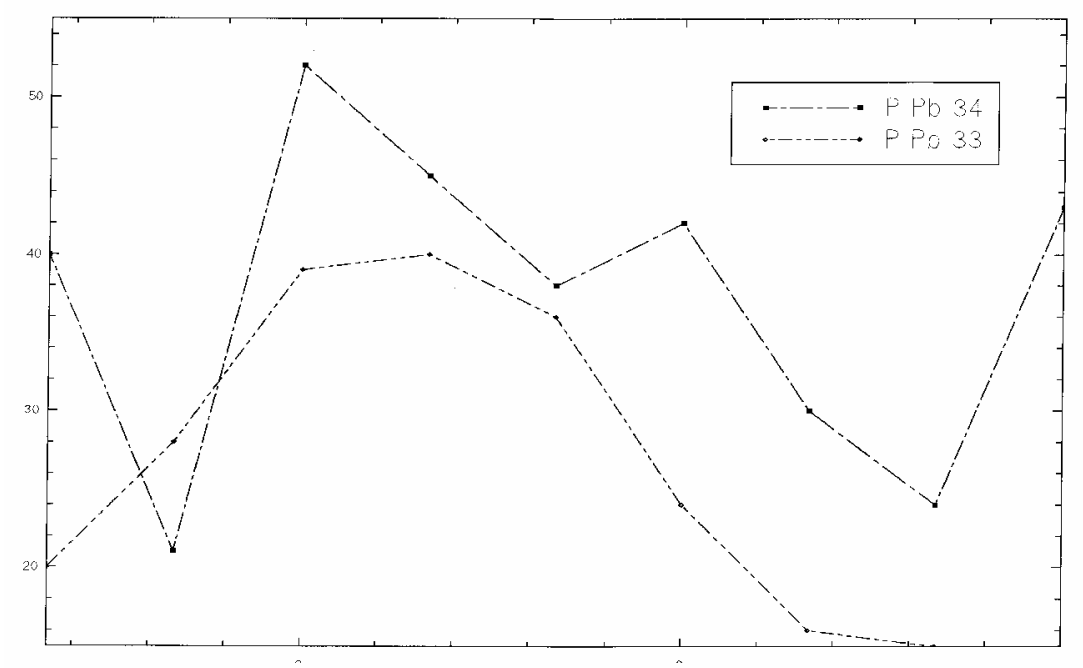

Fig. 8: Correlation between 2 Ss in one SYMLOG dimension (P)

Fig. 8 shows the (typical) correlation in the judgment-dynamics of a student-pair with respect to one dimension $\mathrm{P}$ giving judgments to the shadowy picture.

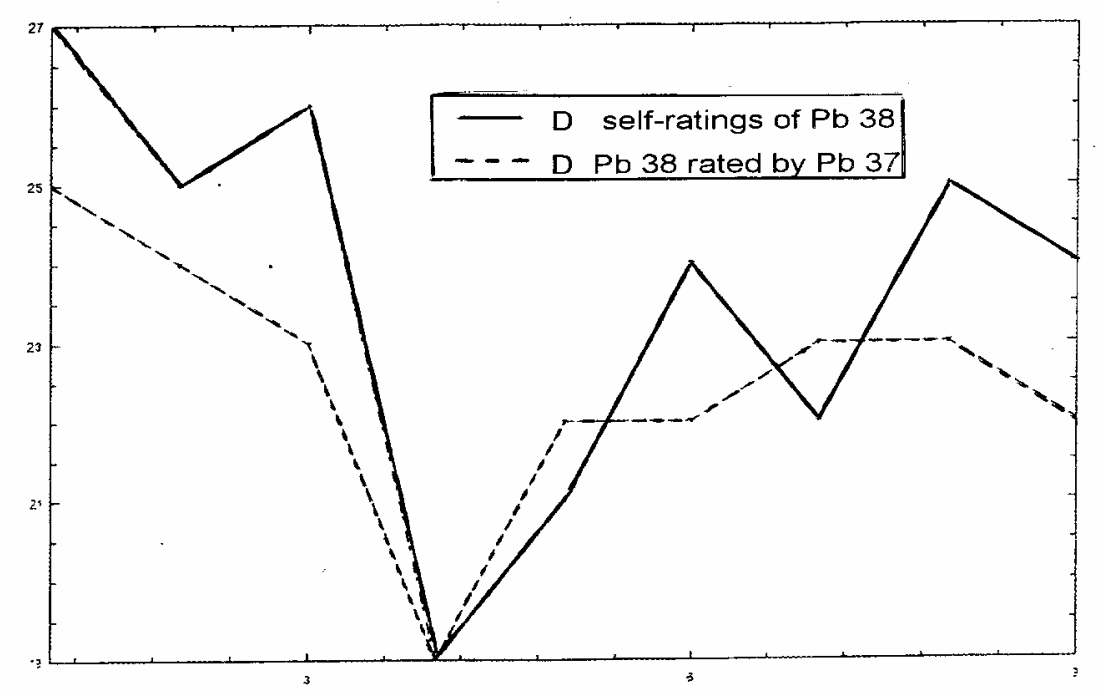

Fig. 9: Correlation between self-ratings of one subject and the ratings given by the partner

Fig. 9 shows the correlation in the judgment-dynamics of a student-pair with respect to one dimension D - however in contrast to Fig. 8 we see the judgments one person gave himself and the judgments the partner gave him (which is not typical but very rare due to the fact that the persons did not give feedback to each other in this aspects). 
The aim of this short and sketchy report of different recent experiments done at Osnabrück is not so much to present the findings and results in some detail. The experiments have to be replicated in order to protect the effects from artificial influences and to go deeper in the analysis of the data. Rather, it was the aim of this report to show how the use of iterative reproductions and the design of experiments from the perspective of pattern formation and completion dynamics leads to systemic effects which are both interesting and worth to carry on further research.

\section{Completion Dynamics, Teleology and Creativity}

For my understanding, the discussed conception of completion dynamics (or pattern formation and pattern recognition due to attracting forces) and the outlined experiments lead to a very fascinating revision of causality. This statement is not only meant from the perspective of the philosophy of science but also from a rather practical perspective - in order to take the process of creativity and imagination more into account.

One of the most inspiring findings and concepts in the field of systems science is the notion of an attractor - the final structure of a process towards which the dynamic tends. So, when we start this process from different initial points (or "situations") it always tends toward the same end-structure (or end-"situation"). With regard to an attractor, the dynamic forces - what "causes" the process to have a special order - are not pushing from behind but pulling from ahead. This is a fascinating and new insight because over the last few centuries it was seldom done in science (and only far away from the mainstream) to think in terms of such a teleological principle, a principle that means accepting forces that pull from in front, from the future, instead of pushing from behind, from the past. Of course, the Aristotelian notion of "entelechy" focuses on such teleological principles; and Goethe, Spinoza and William James have provided the seedbed from which this thinking germinated in the 20th century (including Gestalt psychology). However, until quite recently all these approaches, theories and ways of thinking were far away from being accepted as "scientific" in a strict sense - at least in the area of the natural sciences. The idea that a cause is pulling from ahead and, therefore, is effective from the future seemed to contradict the "normal" principles of causality and, as a consequence, to destroy the foundations of science.

It should be stressed that speaking about forces "pushing from the past" or "pulling from the future" is, of course, not a ontological but a explanatory statement. Therefore, teleology is no more and no less than an principle of explanation - however, this is also true for our classical understanding of causality. As a consequence, teleology and classical causality have the same status.

In order to discuss this point more cleraly, let us go to a statement of the physicist Haken (which we used already in changed manner at the beginning) describing the attracting selforganization of a laser: "When part of the laser atoms are in an ordered state so that they produce a well-defined coherent laser wave, this laser wave acting as an order parameter may enslave the rest of the laser atoms to form a total state in a well-ordered fashion" (Haken 1992: 46). We can see the idea of completion dynamics and the forces of the attractor which "pull from ahead" in a teleological manner. With respect to the dynamics of laser atoms this perspective might be seen too sophisticated or even exaggerated. One could argue that the dynamics could as well be described by using the forces "here and now" - and the term "future" would make no deeper sense. 
Even it I would concede part of this argument in the case of death matter the importance of "forces from the future" come again into our discussion with respect to cognitive and interactive dynamics - especially, with respect to imaginative and creative processes which are, undoubtedly, essentially for man's world. When we ask a student who is going downstairs (in an university building) to a special lecture: "Why are you going?" we would find the explanations very lousy: "due to gravity", or: "due to the impetus of my movement". We also would question the explanation: "because I decided yesterday to go there" - asking, why did he made this decision, what were his expectations? Wouldn't it just be the most simple and satisfying explanation when he say like this: "because I want to hear the lecture of Prof. X"? Here the expectations or imagination of future states are indeed very important in order to understand the dynamics.

It is of interest that this is not only restricted to individual imaginations but can be expanded to the emergence of collective images which orders collective behavior (and by the same way: individual behavior as part of the system dynamics). For example, one can often observe that the chaotic applause which follows a good performance may suddenly and in a self-organized manner become ordered so that hundreds of people are clapping their hands in the same rhythm (sometimes together with some counter-rhythms) which is (for a while) the attractor of the tapping-process. In contrast to the possibility that a leader or teacher in front of the auditorium gives external order by shouting "tap! - tap! - tap!", it should be noticed that this process usually comes to its attractor by self-organization and, therefore, does not require an "organizer". A similar example of an self-organized attractor can be observed when each member of a large group is asked to "listen to" (or imagine) an "inner beat" and/or tone, musical motif, body-movement, etc. and then to express this image of a tap-beat, sound or dancing-movement, more and more. From the chaos of individual beats, tones, motifs and movements emerges a dynamical pattern which stabilizes to a beauty expressive of a common self-organized complex order uniting all the individual images into the manifestation of a group-imagination (at least for a while - however, phase transitions of these patterns may also occur). This can be seen, understood and reconstructed in detail as an attractor of the complex group dynamics.

Let us look a little more carefully at the moment when the attractor establishes itself. To use the much more simple example of an applause-rhythm, one can say that after a period of chaotic applause, the (mostly unconscious) question arises, "What next?" - with respect to the universe of possibilities: going on, stopping the applause, shouting "Bravo!", making some rhythm (due to the fact that many people have experienced this in the past), etc. At this moment the whole situation becomes rather insecure and unstable, and some persons may try to express their image of rhythmic clapping (while others may try to act in another way, doing other things). So, within the chaos of applause, suddenly the rhythmic clapping of some people appears, which again increases the probability that other people will join (again more or less unconsciously) in one or another of these rhythms (in contrast to "normal" individual clapping - which has, of course, a rhythm too - the "rhythmic clapping" usually is more expressive, with larger, more explicit movements, and slower frequency). For a short moment there will be a competition of some of these rhythms. But soon that rhythm most people "prefer"(at this moment - i.e. it is perhaps the best expression of their tacit imagination), will find more and faster others who join that rhythm; this in turn increases the loudness and, with it, the attention of other people, which again increases the probability of joining, and so forth. From the perspective of the finally established attractor, one can say that most people (unconsciously) are "pulled from ahead" by the attracting forces. Accordingly, as long as there is only a part of the group clapping in the rhythm of the final attractor, there is an "appetition for 
completion," as Alfred North Whitehead called this phenomenon: forces which act to complete the whole pattern of the dynamic attractor.

Intentionally, this description of the emergence of the clapping-order was very near to the description of the emergence of order in a laser or in the famous Benard-Instability (where the movement of the molecules is self-organized to big honeycomb cells of movement). However, at least in the case of cognitive processes (and interactive processes influenced by cognition) the idea of "forces from future" by way of imagination and expectation (or, more generally, by creativity) has a deep meaning. As a consequence, I want to plead for a reassessment of and a re-esteem for the teleological principle in order to investigate and understand the ordering forces in human's world.

\section{References}

Ash S (1946) Forming Impressions of Personality. J Abnorm. a. Soc. Psychol, 41: 258-290

Bales RF, Cohen SP (1979) SYMLOG: A System for the Multiple Level Observation of Groups, The Free Press, New York

Bartlett FC (1932) Remembering - A Study in Experimental and Social Psychology. Cambridge Univ. Press

Bröcker T, Kriz J (1999) Formation and Adaptation of Schemata. In: Tschacher W, Dauwalder JP (eds) Dynamics, Synergetics, Autonomous Agents. World Scientific, Singapore, pp 129-142

Ceccini A (1988) Simulation is education. In: Crookall D (ed) Proceedings of the ISAGA $18^{\text {th }}$ Conference 1987. Pergamon Press, New York, pp 213-228

Dörner D, Wearing AT (1995) Complex Problem Solving: Towards a (Computersimulated) Theory.In: Funke J, Frensch P (eds) Complex Problem Solving - The European Perspective. Erlbaum, Hillsdale N.J.

Haken H (1983). Synergetics. An Introduction. Springer, Berlin, New York

Haken H (1988) Neuronal and Synergetic Computers. Springer, Berlin, New York

Haken H (1992) Synergetics in Psychology. In: Tschacher W et al. (eds) Self-Organization and Clinical Psychology. Empirical Approaches to Synergetics in Psychology. Springer, Berlin, New York, pp 32-54

Haken H (1996) Principles of Brain Functioning. A Synergetic Approach to Brain Activity, Behavior and Cognition. Springer, Berlin, New York

Haken H, Kelso J, Bunz, H (1985) A Theoretical Model of Phase Transitions in Human Hand Movement. Biol. Cybernetics, 51:347-356

Haken H, Stadler M (1990) Synergetics of Cognition. Springer, Berlin, New York.

Kim DH (1994) Systems Thinking Tools. Pegasus Communications, Boston

Klabbers J (1989) On the improvement of competence. In: Klabbers J (ed) Proceedings of the ISAGA 19th Conference 1988. Pergamon Press, New York, pp 3-8

Kriz J (1990) Synergetics in clinical psychology. In: Haken H, Stadler M (eds) Synergetics of cognition. Springer, Berlin, New York, pp 393-404

Kriz J (1991) Mental Health: Its Conception in Systems Theory. An Outline of the Person-Centered System Approach. In: Pelaez MJ (ed): Comparative Sociology of Family, Health \& Education, XX, (Malaga, Espania), pp 6061 - 6083

Kriz J (1992) Simulating Clinical Processes by Population Dynamics. . In: Tschacher W et al. (eds) Self-Organization and Clinical Psychology. Springer, Berlin, New York, pp 150-162

Kriz J (1993) Pattern Formation in Complex Cognitive Processes. In: Haken H, Mikhailov A (eds.) Interdisciplinary Approaches to Nonlinear Complex Systems. Springer, Berlin, New York, pp $161-175$ 
Kriz J (1997) On chaos and order. Gestalt Theory, 19: 197 - 212

Kriz WC (1997) Training of Systems-Competence with Gaming Simulations. Conference Proceedings - $28^{\text {th }}$ Conference of the International Simulation And Gaming Association (ISAGA). Tilburg University Press.

Kruse $\mathrm{P}$ et al. (1992) Instability and Cognitive Order Formation: Self-Organization Principles Psychological Experiments and Psychotherapeutic Interventions. In: Tschacher G et al. (eds) Self-Organization and Clinical Psychology. Springer, Berlin, New York, pp 102-117

Meadows D, Toth F (1985) Stratagem-1.A. Microcomputer based management training game on the dynamics of energy/environment interaction

Senge P (1990) The Fifth Discipline. The Art and Practice of the Learning Organization. Currency Doubleday, New York.

Stadler M, Kruse P (1990) The Self-Organization Perspective in Cognition Research: Historical Remarks and New Experimental Approaches. In: Haken H, Stadler M (eds) Synergetics of cognition. Springer, Berlin, New York, pp 32-52

Thiagarajan S (1993) How to maximize transfer from simulation games through systematic debriefing In: Percival F et al. (eds) The Simulation and Gaming Yearbook 1993. Kogan Page, London, pp 45-52.

Tschacher W, Brunner EJ, Schiepek G (1992) (eds) Self-Organization and Clinical Psychology. Empirical Approaches to Synergetics in Psychology. Springer, Berlin, New York

Tschacher W, Dauwalder JP (1999),(eds) Dynamics, Synergetics, Autonomous Agents. Nonlinear Systems Approaches to Cognitive Psychology and Cognitive Science. World Scientific, Singapore 